\title{
Collaboration with high-payload industrial robots: simulation for safety
}

\author{
Beibei Shu $(\bowtie)$, Gabor Sziebig \\ UiT, campus Narvik, Lodve Langesgate 2, 8514 Narvik, Norway \\ e-mail: beibei.shuduit.no
}

\begin{abstract}
When operators and industrial robots are sharing the same task, there are multiple factors, which effect if a system is safe for the human operator or not. In general this is solved by introducing Collaborative Robot for cooperation, but what happens when we would extend our already existing production facilities with newer comfort features?

In this paper we propose to include industrial robots and humans executing the same task, while their safety is supervised by a simulation environment, where all necessary precautions are taken. The task is a simple nut-screw operation, where the industrial robot is executing the hard lifting part for the screw and the human holds the nut to be screwed on the screw. Results are demonstrated through simulation and in reality also.
\end{abstract}

Keywords: Human-Robot Collaboration, industrial robot, simulation, virtual reality

\section{Introduction}

Industry 4.0 related technological developments contributes to sensory systems, which were not available before or were very limited in usability, when we use them in humanrobot collaboration. These sensory systems can be categorized in camera systems, laser range finders, depth cameras or LIDAR systems.

The goal with the usage of the sensory systems is to offer safety/comfort functions to the humans, who supposed to work together with an industrial robots. We can see such examples: augmented reality (AR) based control of industrial robot (1) or force feedback based guidance of industrial robots (2) and (3). However these solutions are providing remote manipulation and not task or space sharing with an industrial robot. There are some experiments carried out with using simulation tools and real-time connection to a physical robot like (4) or (5), but the solutions presented are limited to one type of robot.

Some more advanced solutions, which also include depth cameras and virtual reality can be seen in (6) and (7).

As described in this section with the help of VR simulation, researchers make humanrobot collaboration more flexible and intuitive, and more experiments can be conducted to test different new ideas. 


\section{Proposal on collaboration through virtual reality}

Let us suppose a simple scenario: nut screwing operation, a human holding a nut in one hand and moves the hand towards a detecting area, where a camera is recognizing the human, this is triggered also by a foot switch. A camera/sensor system captures and recognizes the nut then feeds the coordinate of the nut to a central computational unit in real-time. The central computational unit sends the instructions to the robot, and commands the robot to pick up a screw from a box which contains several different objects, and moves the screw to the nut's position with pointing at the centre of the nut hole. And the robot rotates the screw in to the nut as demonstrated in Fig 1.

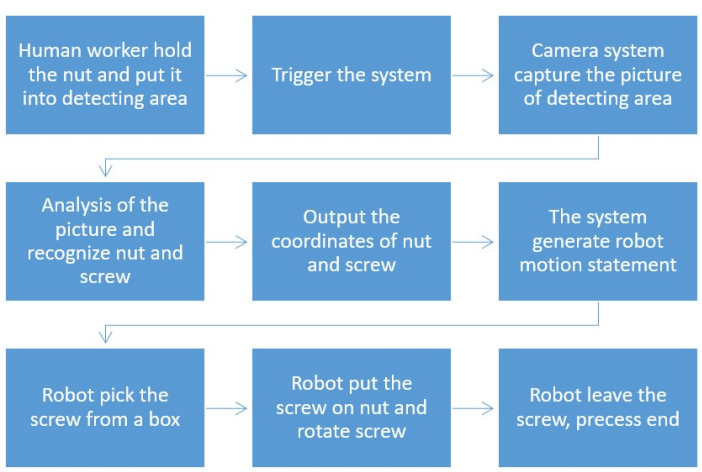

Fig. 1. Sequence flow for nut screwing scenario

This simple nut screwing scenario describes the simplicity of the amount of robot programming. The scenario does not require any knowledge on robot programming, neither on engineering. With such a system, human-robot collaboration can be achieved very effectively and simple.

However today's legislation and safety standards does not allow such interaction between high-payload robots and humans. In order to create a safe environment for the human, we propose to use Virtual Reality solution for such purposes.

According to (8), this task is categorized as Level 4 in Human-Machine Interactionlevels between human and industrial robot. It is called "Shared workspace and shared task with physical interaction". Our goal with the proposal is to achieve this Level 4 interaction with high-payload industrial robots.

\section{Experimenting with collaboration in Virtual Reality}

As proposed in Section 2, a nut screwing experiment was created. The experiment is composed from two parts: a Virtual Realty solution, based on a simulation tool called Visual Components, and an industrial robot produced by NACHI. The simulation tool is running on Windows environment and communicates all necessary information with 
the industrial robot through Ethernet connection, which is a result from previous research project. The simulation tools offers a comprehensive set of tools for programming of industrial robots, which is essential for such kind of experimenting.

An overview of the experiment could be seen in Fig. 2. On the picture we can observer the virtual representations of NACHI MZ-07 industrial robot, the human and the additional equipment, which is necessary for the experiment: a box with nut, screw and ball, camera system and workbench. The experiment starts, with the human holding the nut in his/her hand, which is placed in a random position in the hand. The camera system recognizes the position of all objects, which are visible on the Fig. 2.

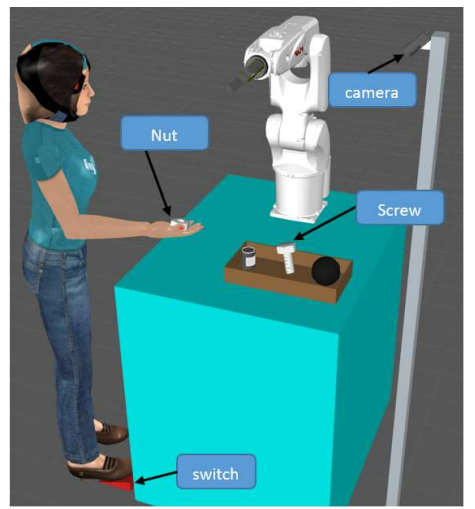

Fig. 2. Overview of the scene for the nut screwing scenario

As the human approaches the industrial robot, the system is responding to the human's proximity as it is described in Section 2. A successful execution of the collaboration is shown in Figure 3.

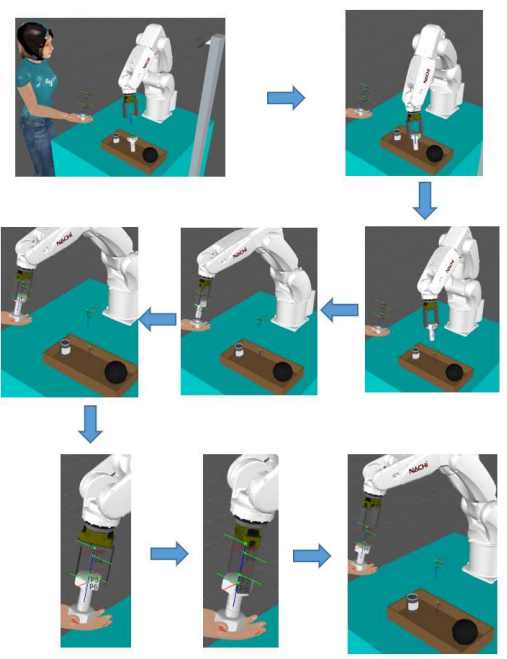

Fig. 3. Execution of the successful collaboration 


\section{Conclusion}

Human-Robot Collaboration is not any more limited to sharing the space with an industrial robot. With the introduction of Virtual Reality, a human worker and a highpayload industrial robot can work on the same task, not only sharing the same space, but also the task itself. In our experiment we show how a simple task of nut screwing operation could be shared between the human operator and the industrial robot. The experiment shows the feasibility of the approach and the Virtual Reality system helps the operator to achieve the necessary comfort functions, which is needed for a Level 4 Human-Machine Interaction.

\section{$5 \quad$ References}

1. Frank JA, Moorhead M, Kapila V, editors. Realizing mixed-reality environments with tablets for intuitive human-robot collaboration for object manipulation tasks. 2016 25th IEEE International Symposium on Robot and Human Interactive Communication (RO-MAN); 2016 26-31 Aug. 2016.

2. Dombrowski U, Stefanak T, Perret J. Interactive Simulation of Human-robot Collaboration Using a Force Feedback Device. Procedia Manufacturing. 2017;11:12431.

3. Ando, Noriaki, Peter Tamas Szemes, Peter Korondi, and Hideki Hashimoto. Friction compensation for 6dof cartesian coordinate haptic interface. In Intelligent Robots and Systems, 2002. IEEE/RSJ International Conference on, vol. 3, pp. 2893-2898. 2002.

4. de Giorgio A, Romero M, Onori M, Wang L. Human-machine Collaboration in Virtual Reality for Adaptive Production Engineering. Procedia Manufacturing. 2017;11:1279-87.

5. Gammieri L, Schumann M, Pelliccia L, Di Gironimo G, Klimant P. Coupling of a Redundant Manipulator with a Virtual Reality Environment to Enhance Humanrobot Cooperation. Procedia CIRP. 2017;62:618-23.

6. Matsas E, Vosniakos G-C, Batras D. Prototyping proactive and adaptive techniques for human-robot collaboration in manufacturing using virtual reality. Robotics and Computer-Integrated Manufacturing. 2018;50:168-80.

7. Moniri MM, Valcarcel FAE, Merkel D, Sonntag D, editors. Human Gaze and Focus-of-Attention in Dual Reality Human-Robot Collaboration. 2016 12th International Conference on Intelligent Environments (IE); 2016 14-16 Sept. 2016.

8. Bdiwi M, Pfeifer M, Sterzing A. A new strategy for ensuring human safety during various levels of interaction with industrial robots. CIRP Annals. 2017;66(1):453-6. 